\title{
Sprachtherapie für Sprachstörungen nach einem Schlaganfall
}

Brady MC, Kelly H, Godwin J et al. Speech and language therapy for aphasia following stroke. Cochrane Database Syst Rev 2016; 6: CD000425. DOI 10.1002/14651858.CD000425.pub4

Eine Aphasie ist eine erworbene Sprachstörung, die durch Hirnschädigung verursacht wird. Sie kann einzelne oder alle sprachlichen Modalitäten betreffen: Sprechen und Verstehen sowie Lesen und Schreiben. Etwa ein Drittel aller Personen erleidet als Folge eines Schlaganfalls eine Aphasie.

\section{Ziele}

Untersuchung der Wirksamkeit von Aphasietherapie nach Schlaganfall.

\section{Literatursuche}

Wir durchsuchten das Cochrane Stroke Group Trials Register (letzte Suche am 9. September 2015), CENTRAL (2015, 5. Ausgabe) und weitere Cochrane Library Datenbanken (CDSR, DARE, HTA, bis 22. September 2015), MEDLINE (1946 bis September 2015), EMBASE (1980 bis September 2015), CINAHL (1982 bis September 2015), AMED (1985 bis September 2015), LLBA (1973 bis September 2015) und SpeechBITE (2008 bis September 2015). Auch durchsuchten wir die wichtigsten Studienregister für laufende Studien wie ClinicalTrials.gov (bis 21. September 2015), Stroke Trials Registry (bis 21. September 2015), Current Controlled Trials (bis 22. September 2015) und WHO ICTRP (bis 22. September 2015). Um weitere veröffentlichte, unveröffentlichte und laufende Studien zu finden, suchten wir händisch im International Journal of Language and Communication Disorders (1969 bis 2005) sowie in Literaturverzeichnissen relevanter Artikel und kontaktierten akademische Institutionen und andere Wissenschaftler. Hinsichtlich der Sprache der Artikel gab es keine Einschränkungen.

\section{Auswahlkriterien}

Randomisierte kontrollierte Studien (RCTs), die Sprachtherapie (formale Intervention mit dem Ziel die Sprach- und Kommunikationsfähigkeit sowie Aktivität und Teilhabe zu verbessern) mit Nicht-Behandlung, mit sozialer Unterstützung bzw. Stimulierung (Interven- tion, die soziale Unterstützung bietet und die Kommunikation stimuliert, aber keine gezielten therapeutischen Interventionen beinhaltet) oder mit einer anderen sprachtherapeutischen Interventionsform (die sich hinsichtlich Dauer, Intensität, Frequenz, Behandlungsmethode oder theoretischem Ansatz unterscheidet) vergleichen.

\section{Datenerhebung und -analyse}

Unabhängig voneinander extrahierten wir die Daten und bewerteten die Qualität der eingeschlossenen Studien. Fehlende Daten erfragten wir bei den Studiendurchführenden.

\section{Wesentliche Ergebnisse}

Wir schlossen 57 RCTs (74 randomisierte Vergleiche) mit insgesamt 3002 Teilnehmern in dieses Review ein (wobei einige Teilnehmer in mehreren Vergleichen vorkamen). 27 randomisierte Vergleiche (1620 Teilnehmer) untersuchten Sprachtherapie versus Nicht-Behandlung; Sprachtherapie führte zu klinisch und statistisch signifikanten Verbesserungen der funktionalen Kommunikation der Patienten (standardisierte Mittelwertdifferenz (SMD) 0.28, 95\% Konfidenzintervall (KI) 0.06 bis $0.49, \mathrm{P}=0.01$ ), des Lesens und Schreibens sowie der expressiven Sprache. Im Follow-up waren diese Verbesserungen (aufgrund verringerter Stichprobengrößen) jedoch nicht mehr nachweisbar. Neun randomisierte Vergleiche (447 Teilnehmer) untersuchten Sprachtherapie versus soziale Unterstützung und Stimulierung; Metaanalysen erbrachten keine Evidenz für Unterschiede hinsichtlich der funktionalen Kommunikation, jedoch brachen mehr Teilnehmer die Therapie mit sozialer Unterstüt-

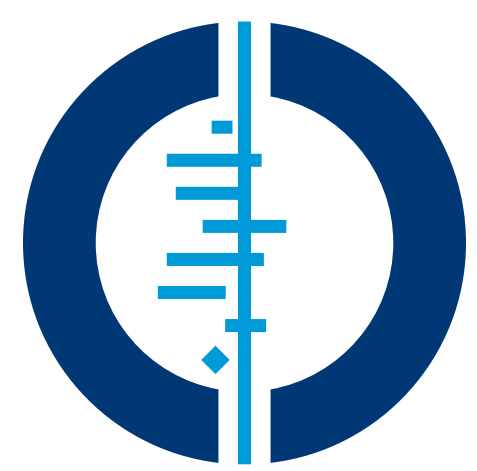

zung ab als jene mit Sprachtherapie. 38 randomisierte Vergleiche (1242 Teilnehmer) verglichen zwei sprachtherapeutische Ansätze. Menschen mit Aphasie, die Therapie mit hoher Intensität, hoher Gesamtstundenzahl und über einen längeren Zeitraum hinweg erhielten, zeigten eine signifikant bessere Kommunikationsfähigkeit als Menschen, die Therapie mit einer niedrigeren Intensität, einer niedrigeren Gesamtstundenzahl oder über einen kürzeren Zeitraum hinweg erhielten. Der Nutzen einer hohen Therapieintensität oder hohen Gesamtstundenzahl wurde durch eine signifikant höhere Drop-Out-Rate in diesen Therapiegruppen eingeschränkt. Generell wurden in den Studien wenige Teilnehmer mit unterschiedlichsten Merkmalen (Alter, Zeit seit Schlaganfall, Schweregradprofile), Interventionen und Therapieergebnissen randomisiert.

\section{Schlussfolgerungen der Autoren}

Unser Review stellt Evidenz zur Wirksamkeit von Sprachtherapie bei Menschen mit Aphasie nach Schlaganfall in Form von Verbesserungen der funktionalen Kommunikation, des Lesens und Schreibens sowie der expressiven Sprache im Vergleich zu Nicht-Behandlung bereit. Es gibt Hinweise darauf, dass Therapie mit hoher Intensität, hoher Gesamtstundenzahl oder über einen längeren Zeitraum hinweg förderlich sein kann. Therapien mit hoher Intensität oder Gesamtstundenzahl werden möglicherweise nicht von allen Teilnehmern angenommen.

\section{Übersetzung:}

A. de Sunda, F. Krzok, B. Wellner, freigegeben durch Cochrane Deutschland. 


\section{Kommentar}

Das aktuelle Update der Cochrane-Metaanalyse „Sprachtherapie bei Aphasie nach Schlaganfall“ bewertet die vorliegende Evidenz für die Wirksamkeit von Sprachtherapie auf die sprachlichen und kommunikativen Fähigkeiten von Menschen mit Aphasie nach Schlaganfall. Verglichen mit der vorherigen Version aus 2012 ist die Zahl der in die Metaanalyse eingeschlossenen randomized controlled trials (RCT) zwischen 2011 und 2015 von 39 auf 57 gestiegen, was auf eine international stark ansteigende Forschungstätigkeit zum Thema Aphasie schließen lässt.

Für die aktuelle Metaanalyse wurde der zentrale Aspekt der Therapieinhalte mit einem neuen detaillierten Beschreibungsraster für sprachtherapeutische Interventionen, der sogenannten TIDieRCheckliste [1,2], analysiert. Diese Checkliste setzt neue Standards für die Genauigkeit, mit der Forschungsergebnisse zur Aphasietherapie berichtet werden (sollen), indem sie explizit Rahmenbedingungen der Therapie wie Ziele,
Materialien, Vorgehensweisen oder individuelle Modifikationen abfragt.

Im Vergleich zur letzten Version fallen die Schlussfolgerungen der Autoren jetzt zuversichtlicher aus. War 2012 noch vorsichtig von „some evidence“ (einigen Hinweisen) für die Wirksamkeit von Aphasietherapie die Rede, stellen die Autoren jetzt eindeutig einen Vorteil der Aphasietherapie (gegenüber der NichtBehandlung) fest. Laut den Autoren sind vermutlich vor allem hochintensive Therapieformen wirksam. Es fehlen jedoch vor allem Daten zur Stabilität des Therapieerfolgs. Auch wird nach Ansicht der Autoren noch zu wenig über Auswirkungen der Therapie auf die Partizipation und Lebensqualität von Menschen mit Aphasie berichtet.

Diese offenen Fragen werden zum Teil von einem noch nicht in dieses Update aufgenommenen neuen RCT beantwortet [3], der langanhaltende Verbesserungen von Kommunikationsfähigkeit und Lebensqualität nach intensiver Aphasietherapie bei über 150 Teilnehmern mit chronischer Aphasie zeigt.
Die Autorin

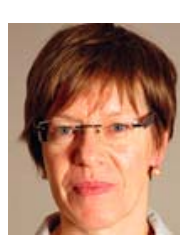

Prof. Annette Baumgärtner PhD, Hochschule Fresenius, Fachbereich Gesundheit \& Soziales, Hamburg

\section{Literatur}

1. Hoffmann T, Glasziou P, Boutron I et al. Better reporting of interventions: template for intervention description and replication (TIDieR) checklist and guide. BMJ 2014; 348: g1687

2. Voigt-Radloff S, A. Blümle A, Meerpohl J. Die TIDieR Checkliste und Anleitung - ein Instrument für eine verbesserte Interventionsbeschreibung und Replikation. Gesundheitswesen 2016; 78: 175-188

3. Breitenstein C, Grewe T, Flöel A et al. Intensive speech and language therapy in patients with chronic aphasia after stroke: a randomised, open-label, blinded-endpoint, controlled trial in a health-care setting. Lancet 2017; 389: 1528-1538. DOI: http://dx.doi.org/10.1016/ S0140-6736(17)30067-3 